\title{
Blockade of hypoxia-induced CXCR4 with AMD3100 inhibits production of OA-associated catabolic mediators IL-1 $\beta$ and MMP-13
}

\author{
PENGCUI LI $^{1 *}$, JIN DENG $^{2 *}$, XIAOCHUN WEI ${ }^{1}$, CHATHURAKA T. JAYASURIYA ${ }^{3}$, \\ JINGMING ZHOU ${ }^{3}$, QIAN CHEN $^{3}$, JIANZHONG ZHANG ${ }^{4}$, LEI WEI ${ }^{1,3}$ and FANGYUAN WEI ${ }^{4 *}$ \\ ${ }^{1}$ Department of Orthopaedics, The Second Hospital of Shanxi Medical University, Shanxi Key Laboratory \\ of Bone and Soft Tissue Injury Repair, Taiyuan, Shanxi 030001; ${ }^{2}$ Department of Emergency, The Affiliated Hospital \\ of Guizhou Medical University, Guiyang, Guizhou 550004, P.R. China; ${ }^{3}$ Department of Orthopaedics,
} Warren Alpert Medical School of Brown University, Providence, RI 02903, USA; ${ }^{4}$ Foot and Ankle Orthopaedic Surgery Center, Beijing Tongren Hospital, Capital Medical University, Beijing 100730, P.R. China

Received August 21, 2015; Accepted May 5, 2016

DOI: $10.3892 / \mathrm{mmr} .2016 .5419$

\begin{abstract}
Binding of the chemokine stromal cell-derived factor-1 (SDF-1) to its receptor C-X-C chemokine receptor type 4 (CXCR4) results in receptor activation and the subsequent release of matrix metalloproteinases (MMPs) that contribute to osteoarthritis (OA) cartilage degradation. As hypoxia is a defining feature of the chondrocyte microenvironment, the present study investigated the possible mechanism through which SDF-1 induces cartilage degradation under hypoxic conditions. To do this, OA chondrocyte cultures and patient tissue explants pretreated with the CXCR4 inhibitor, AMD3100 were incubated with SDF-1. It was identified that hypoxic conditions significantly elevated the expression of CXCR4 in osteoarthritic chondrocytes relative to normoxic conditions. Furthermore, SDF-1 elevated MMP-13 mRNA levels and proteinase activity. It also elevated the mRNA and protein levels of runt-related transcription factor 2 , and induced the release of glycosaminoglycans and the inflammatory cytokine, interleukin-1 $\beta$. By contrast, such changes did not occur to an appreciable degree in cells that were pretreated with AMD3100. The results of the present study demonstrate that even under hypoxic conditions, where CXCR4 expression is significantly elevated in chondrocytes, AMD3100
\end{abstract}

Correspondence to: Dr Fangyuan Wei, Foot and Ankle Orthopaedic Surgery Center, Beijing Tongren Hospital, Capital Medical University, 1 Dongjiaoming Road, Beijing 100730, P.R. China

E-mail: footwfy@126.com

*Contributed equally

Key words: stromal cell-derived factor-1, C-X-C chemokine receptor type 4, chondrocytes, cartilage degradation, hypoxia, AMD3100 effectively blocks this receptor and protects chondrocytes from OA-induced catabolism, suggesting that the successful inhibition of CXCR4 may be an effective approach for OA treatment.

\section{Introduction}

Osteoarthritis (OA) is the most common degenerative joint disease among populations that are past middle age (1). In Asian countries, the incidence of OA in individuals aged $>65$ years, will increase from $6.8 \%$ in 2008 to $16.2 \%$ in 2040 (2). Although the exact mechanism underlying OA pathogenesis remains unclear, OA is largely defined by cartilage tissue breakdown resulting from a dysregulated process of tissue homeostasis that is crucial to this degenerative joint disease. Elevated levels of catabolic proteases, such as the matrix metalloproteinases (MMPs) and inflammatory cytokines, such as interleukin-1 $\beta$ (IL-1 $\beta$ ) and tumor necrosis factor $\alpha$ (TNF- $\alpha)$ contribute significantly to the breakdown of the cartilage extracellular matrix (ECM) (3-7). The resulting cleavage and release of matrix molecules, such as glycosaminoglycans (GAGs), type II collagen and hyaluronan contribute to the overall loss of healthy cartilage tissue $(3,7)$. Furthermore, cleavage fragments generated from the catabolism of these ECM molecules exacerbate disease conditions by promoting further tissue destruction (7).

While many members of the pool of catabolic mediators that are involved with OA pathogenesis have been the subjects of extensive investigation, stromal cell-derived factor-1 (SDF-1) remains relatively less well-known. SDF-1 is an 8 -kDa peptide that regulates cellular activities via interactions with $\mathrm{C}-\mathrm{X}-\mathrm{C}$ chemokine receptor type 4 (CXCR4) found on chondrocytes (8). Synovial fluid SDF-1 concentrations are markedly elevated in rheumatoid arthritic and osteoarthritic joints (9). Furthermore, pathological concentrations of SDF-1 induce human chondrocyte death in vitro (10). Binding of SDF-1 to CXCR4 on chondrocytes results in the release of the OA-associated catabolic proteases, MMP-3, -9 and -13 (11). 
However, the mechanism by which CXCR4 is regulated in chondrocytes remains to be elucidated. Previous studies have demonstrated that runt-related transcription factor 2 (Runx2) regulates MMP-13 expression (12). Increased Runx2 has been found in OA cartilage, which contributes to the increased expression of MMP-13 in human OA chondrocytes (13). Recently, Zhu et al (14) demonstrated that pretreatment of a pluripotent mesenchymal $\mathrm{C} 2 \mathrm{C} 12$ cell line with SDF-1 siRNA for 2 days led to a marked decrease in Runx 2 protein concentration. The inhibitory effect of SDF-1 siRNA was largely reversed by the addition of excess recombinant SDF-1, suggesting that SDF-1 signaling may regulate Runx 2 expression.

In attempting to improve understanding of the pathophysiology of OA with respect to articular cartilage, it is critical to recognize that cartilage is inherently avascular and, as such, has significantly lower levels of oxygen (hypoxic) than many other tissue types (15-16). Hypoxia often acts as a regulator of certain molecular markers and thereby alters specific cellular pathways (17). Hypoxia has been demonstrated to regulate CXCR4 expression in normal and tumor cells (18-19). It is therefore likely that hypoxia also regulates CXCR4 expression in chondrocytes. The molecular mechanism underlying the hypoxic regulation of the SDF-1/CXCR4 signaling pathway in OA chondrocytes remains to be elucidated. An improved understanding of this process may result in novel strategies for pharmacological intervention in OA. In the present study, the effect of hypoxia on CXCR4 expression in OA chondrocytes was investigated to elucidate a mechanism through which SDF-1 induces cartilage degradation. In addition, the efficacy of the commercially available CXCR4 inhibitor, AMD3100 (20) in reducing OA chondrocyte catabolism under hypoxic conditions was investigated.

\section{Materials and methods}

Chondrocyte isolation and primary culture. Cartilage was obtained from patients undergoing total knee replacement surgery at the Second Hospital of Shanxi Medical University (Taiyuan, China) between September 2012 and December 2013. Cartilage samples were removed from the tibia plateau and washed in Gibco Dulbecco's modified Eagle's medium (DMEM; Thermo Fisher Scientific, Inc., Waltham, MA, USA). Chondrocytes were isolated from the cartilage as previously described (21). Briefly, small samples of cartilage were minced, digested with $2 \mathrm{mg} / \mathrm{ml}$ pronase (Roche Diagnostics, Basel, Switzerland) in Gibco Hank's balanced salt solution (HBSS; Thermo Fisher Scientific, Inc.) for $30 \mathrm{~min}$ at $37^{\circ} \mathrm{C}$, and washed with DMEM. Cartilage samples were digested with $1 \mathrm{mg} / \mathrm{ml}$ bacterial collagenase (Type IA; Sigma-Aldrich, St. Louis, MO, USA) for $6-8 \mathrm{~h}$ at $37^{\circ} \mathrm{C}$ in a shaker. The enzymatic reaction was terminated with DMEM containing $10 \%$ fetal bovine serum (FBS; Gibco; Thermo Fisher Scientific, Inc.). Residual multicellular aggregates were removed by filtration and the cells were washed three times in DMEM. Chondrocytes were plated in DMEM containing 10\% FBS, Invitrogen L-glutamine (2.5 mM; Thermo Fisher Scientific, Inc.), and antibiotics (100 U/ml penicillin; $0.1 \mathrm{mg} / \mathrm{ml}$ streptomycin). Once cells reached confluence, they were split once (passage 1) and grown to confluence again. Cells were plated in 8-well chambers (Nalge Nunc International Corp, Naperville, IL,
USA) at $1 \times 10^{5}$ cells/well or in $100-\mathrm{mm}$ diameter culture dishes (BD Biosciences, Franklin Lakes, NJ, US) at $1 \times 10^{6}$ cells/plate. At $70 \%$ confluence, certain cells were treated with $5 \mathrm{ng} / \mathrm{ml}$ AMD3100 (ApexBio, Boston, MA, USA), 2 h prior to SDF-1 (GenScript, Piscataway, NJ, USA) incubation. Cells were then cultured under hypoxic condition $\left(2 \% \mathrm{O}_{2}\right)$ for two days. Cells $\left(5 \times 10^{6}\right)$ were treated with SDF-1 only $(40 \mathrm{ng} / \mathrm{ml})$ and control cells were cultured in the absence of SDF-1. Cells were incubated in the culture medium for two days and the chondrocyte culture medium was replaced with fresh medium without hyaluronidase. Cells were incubated at $37^{\circ} \mathrm{C}$ in 2 or $5 \% \mathrm{O}_{2}$.

Cartilage explant organ culture. Cartilage explants of uniform size $(1.0 \times 1.0 \times 0.2 \mathrm{~cm})$ were obtained from patients undergoing total knee replacement surgery. Each explant was divided into three segments. Explants were cultured at $2 \% \mathrm{O}_{2}$ to mimic hypoxic conditions, as previously described $(22,23)$. One segment was incubated with AMD3100 (5 ng/ml) prior to SDF-1 $(40 \mathrm{ng} / \mathrm{ml})$ treatment, one segment was incubated with SDF-1 (40 ng/ml) only and the third segment was cultured in the absence of SDF-1 in DMEM containing $10 \% \mathrm{FBS}$ at $37^{\circ} \mathrm{C}$. The cartilage and conditioned media were collected two days subsequently.

Immunocytochemistry. Following a two-day incubation, cells cultured in 8 -well chambers were fixed at $-20^{\circ} \mathrm{C}$ with $70 \%$ ethanol and $50 \mathrm{mM}$ glycine ( $\mathrm{pH} 2.0)$ for $20 \mathrm{~min}$. Slides were then washed with phosphate-buffered saline (PBS) and incubated with a monoclonal anti-CXCR4 antibody at 1:500 (cat. no. mab173; R\&D Systems, Inc., Minneapolis, MN, USA) for $1 \mathrm{~h}$ at $37^{\circ} \mathrm{C}$ followed by incubation with a rhodamine-labeled secondary antibody at 1:1,000 (cat. no. NL557; R\&D Systems, Inc.) for $1 \mathrm{~h}$ at room temperature. Hoechst $(0.5 \mathrm{mg} / \mathrm{ml})$, a florescent nuclear binding dye, was applied following washing with PBS. Slides were then washed and mounted in $95 \%$ glycerol in PBS. Single or multiple exposure photography was performed with a Nikon E800 microscope and Nikon Capture NX2 v2.4.7 (Nikon Inc., Melville, NY, USA).

Quantification of GAGs. To determine the concentration of GAG released by the cultured cartilage explants, the incubation media of the explants were collected following AMD3100 and/or SDF-1 treatment, and GAG concentrations were quantified spectrophotometrically by the 1,9-dimethylmethylene blue (DMMB) assay, using bovine chondroitin sulfate (Santa Cruz Biotechnology, Inc., Dallas, Texas, USA) as a standard, according to a previous study (24).

Western blot analysis. Total protein was extracted from cells and quantified using the BCA Protein Assay Reagent kit (Thermo Fisher Scientific, Inc., Waltham, MA, USA) according to the manufacturer's instructions. Subsequently, $40 \mu \mathrm{g}$ total protein was separated by electrophoresis $(110 \mathrm{~V}$ for $2 \mathrm{~h}$ ) on a $10 \%$ SDS-PAGE gel before being transferred to a nitrocellulose membrane. To detect hypoxia-inducible factor $1 \alpha(\mathrm{HIF}-1 \alpha)$ protein, the membrane was probed with a rabbit anti-human HIF-1 $\alpha$ antibody (cat. no. 3716; Cell Signaling Technology, Inc., Danvers, MA, USA). The antibody was diluted 1:1,000 in blocking buffer (LI-COR Biosciences, Lincoln, NE, USA). Goat anti-rabbit IRDye 680 IgG (cat. no. 
926-68,071; LI-COR Biosciences) diluted 1:5,000 in blocking buffer (LI-COR Biosciences) served as the secondary antibody. To detect Runx 2 protein, the membrane was incubated with a rabbit anti-human Runx2 polyclonal antibody (cat. no. bs-1134R; Bioss, Inc., Woburn, MA, USA) diluted 1:1,000 in blocking buffer. Goat anti-rabbit IRDye $800 \mathrm{CW} \mathrm{IgG} \mathrm{at}$ 1:8,000 (cat. no. 926-32,211; LI-COR Biosciences) served as the secondary antibody. The blots were detected using the Odyssey Infrared Imaging system (LI-COR Biosciences). To ensure even loading of samples, the same membrane was probed with a goat anti-human $\beta$-actin polyclonal antibody (cat. no. SC-1616; Santa Cruz Biotechnology, Inc.) at a dilution of 1:1,000. The relative intensities of western blot bands were semi-quantified by densitometry following normalization to b-actin using ImageJ software (National Institutes of Health; $\mathrm{NIH}$, Bethesda, MA, USA) as previously described (25).

Reverse transcription-quantitative polymerase chain reaction $(R T-q P C R)$. RT-qPCR was performed as previously reported (10). Briefly, total RNA was isolated from the chondrocytes with the Qiagen RNeasy isolation kit (Qiagen, Inc., Valencia, CA, USA). A total of $1 \mu \mathrm{g}$ RNA was transcribed into cDNA using iScripTM (Bio-Rad Laboratories, Inc., Hercules, CA, USA). The resulting cDNA (40 ng/ml) served as the template to quantify the relative content of mRNA using a QuantiTect SYBR Green PCR kit (Qiagen, Inc.) with DNA Engine Opticon 2 Continuous Fluorescence Detection system. (MJ Research, Waltham, MA, USA). The amplification conditions were as follows: Pre-incubation, 2 min at $50^{\circ} \mathrm{C}$; enzyme activation, $10 \mathrm{~min}$ at $95^{\circ} \mathrm{C}$; denaturation, 40 cycles of $95^{\circ} \mathrm{C}$ for $10 \mathrm{sec}$; annealing, $55^{\circ} \mathrm{C}$ for $30 \mathrm{sec}$; and extension, $72^{\circ} \mathrm{C}$ for $30 \mathrm{sec}$. The primers were designed using Primers Express software v1.0 (BioTools Inc., Edmonton, AB, Canada) and were as follows: Forward, 5'-AAACTGAGAAGCATG ACGGACAA-3' and reverse, 5'-GCCAACATAGACCAC CTTTTCAG-3' for CXCR4; forward, 5'-TGCTGCATTCTC CTTCAGGA-3' and reverse, 5'-ATGCATCCAGGGGTC CTGGC-3' for MMP-13; and forward, 5'-CTTCGCCGTCCA TTCACTCC-3' and reverse, 5'-GTGCATTCGTGGGTTGGA GA-3' for Runx2. The 18S ribosomal RNA (rRNA; forward, 5'-CGGCTACCACATCCAAGGAA-3' and reverse, 5'-GCT GGAATTACCGCGGCT-3') was amplified at the same time and served as an internal control. The cycle threshold values for 18S rRNA, CXCR4, MMP-13 and Runx2 were measured and calculated by IQ5 Optical System software, version 2.1 (Bio-Rad Laboratories, Inc.).). Relative transcript levels were calculated as $\mathrm{x}=2-\Delta \Delta^{\mathrm{Cq}}$, in which $\Delta \Delta \mathrm{Cq}=\Delta \mathrm{E}-\mathrm{DC}$, and $\Delta \mathrm{E}=\mathrm{Cq}_{\mathrm{exp}}-\mathrm{Cq}_{18 \mathrm{~s}} ; \Delta \mathrm{C}=\mathrm{Cq}_{\text {control }}-\mathrm{Cq}_{18 \mathrm{~s}}(26)$.

ELISA. MMP-13 activity in conditioned media was measured using a double-antibody sandwich ELISA kit (MMP-13 ELISA kit; GE Healthcare Life Sciences, Chalfont, UK). Assay Diluent RD1-55 (100 $\mu$ l) was added to each well with $100 \mu \mathrm{l}$ standard or sample. The wells were covered with an adhesive strip and incubated for $2 \mathrm{~h}$ at room temperature on a horizontal orbital microplate shaker set at $500 \mathrm{rpm}$. Each well was aspirated and washed three times with $400 \mu \mathrm{l}$ wash buffer. A total of $200 \mu 1$ MMP-13 conjugate was added to each well, covered with a fresh adhesive strip, and incubated for $2 \mathrm{~h}$ at room temperature on the shaker. The aspiration and washing steps were repeated and $200 \mu 1$ substrate solution was added to each well and incubated for $30 \mathrm{~min}$ at room temperature in the dark. Stop solution $(50 \mu \mathrm{l})$ was added to each well and the optical density of each well was determined within 30 min using a microplate reader (Packard FluoroCount BF10000) set at $450 \mathrm{~nm}$. IL-1 $\beta$ in conditioned media collected from cartilage explants was measured using an IL-1 $\beta$ ELISA kit from Invitrogen (cat. no. KMC0011C; Thermo Fisher Scientific, Inc.). Culture medium $(50 \mu \mathrm{l})$ was mixed with $50 \mu \mathrm{l}$ incubation buffer and $50 \mu \mathrm{l}$ biotinylated anti-IL-1 $\beta$ (cat. no. KMC0011C; Thermo Fisher Scientific, Inc.) solution in the wells. Following incubation at $37^{\circ} \mathrm{C}$ for $90 \mathrm{~min}, 100 \mu \mathrm{l}$ streptavidin-horseradish peroxidase working solution was added to the wells and incubated at room temperature for $30 \mathrm{~min}$. The wells were washed with washing solution four times. Following the addition of $100 \mu \mathrm{l}$ stabilized chromogen to each well, the plate was placed in the dark for $30 \mathrm{~min}$ at room temperature. Stop solution $(100 \mu \mathrm{l})$ was then added and the absorbance was recorded at $450 \mathrm{~nm}$. All samples were run in duplicate.

Statistical analysis. All quantitative data were expressed as means \pm standard deviation, obtained from at least three independent experiments and analyzed with SPSS software (version 20.0; IBM SPSS, Armonk, NY, USA). Independent student's $t$ test was used for RT-qPCR quantification of CXCR4 expression in chondrocytes; one-way analysis of variance with Tukey's post-hoc test was used for group comparisons in all other experiments. $\mathrm{P}<0.05$ was considered to indicate a statistically significant difference.

\section{Results}

Hypoxia enhances CXCR4 expression in human OA chondrocytes. Consistent with previously established methods $(22,23)$, $\mathrm{OA}$ articular chondrocytes were maintained in $5 \% \mathrm{O}_{2}$ (normoxia) or exposed to $2 \% \mathrm{O}_{2}$ (hypoxia) for two days. RT-qPCR analysis demonstrated that CXCR4 expression was significantly higher in OA chondrocytes cultured under hypoxic compared with normoxic conditions $(\mathrm{P}=0.006$; Fig. 1A and B). Protein quantification of HIF-1 $\alpha$ via western blotting was performed to confirm that conditions were hypoxic in the in vitro model ( $\mathrm{P}=0.02$; Fig. $1 \mathrm{C}$ and $\mathrm{D})$. Micrographs of immunocytochemical analysis further identified strong CXCR4 positive staining in hypoxic conditions compared with normoxic conditions (Fig. 2).

SDF-1 increases MMP-13 expression level and activity in human OA chondrocyte. The conditioned media were collected for ELISA analysis $48 \mathrm{~h}$ following treatment with SDF-1. As presented in Fig. 3A, SDF-1 (incubated at concentrations of 10-80 ng/ml) increased MMP-13 activity in a dose-dependent manner. Notably, the effect of SDF-1 was greatest at a dosage of $40 \mathrm{ng} / \mathrm{ml}$. RT-qPCR and ELISA results indicated that SDF-1 increased the expression level of MMP-13 ( $\mathrm{P}=0.009$; Fig. 3B), while suppression of MMP-13 enzyme activity was observed by blocking the SDF-1 pathway with AMD3100 ( $\mathrm{P}=0.001$; Fig. 3C).

SDF-1 increases Runx2 expression levels in human cartilage chondrocytes. Total RNA and cell lysates were collected 
A
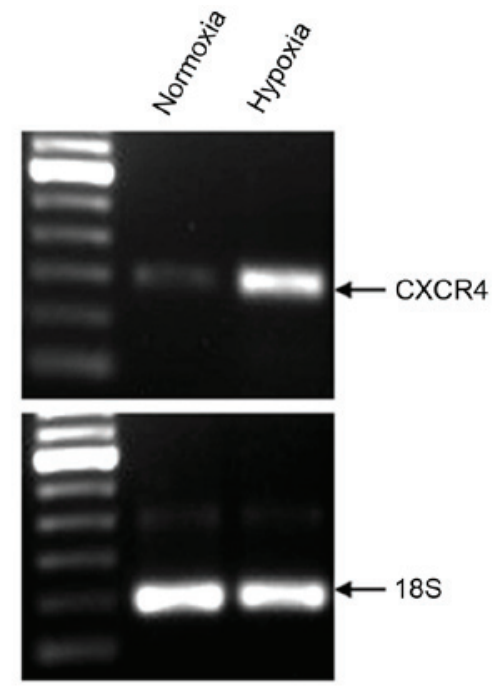

$\mathrm{C}$

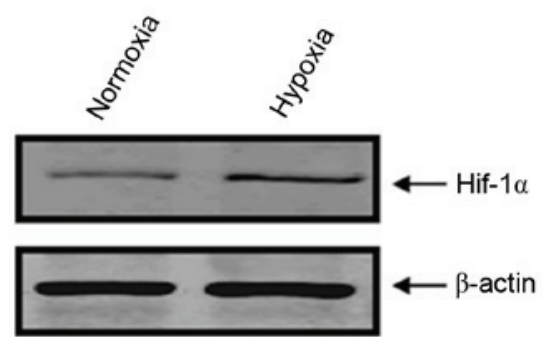

B

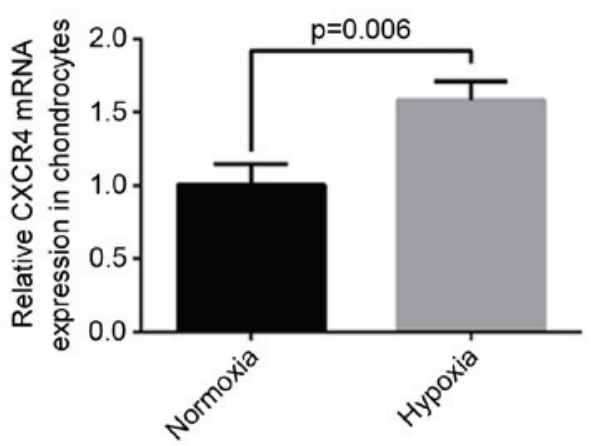

$\mathrm{D}$

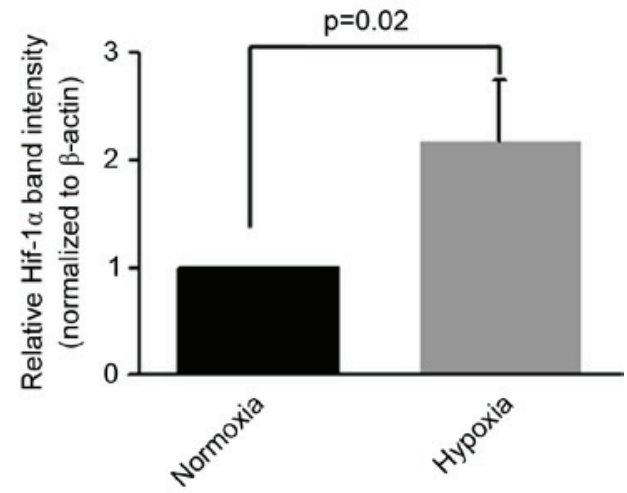

Figure 1. Hypoxia increases CXCR4 expression in human OA chondrocytes. (A and B) Quantitative analysis of CXCR4 gene expression by reverse transcription-quantitative polymerase chain reaction. (C) Western blot of HIF-1 $\alpha$ in OA chondrocytes cultured under normoxic or hypoxic conditions $(5$ or $2 \%$ oxygen, respectively). (D) Band intensities obtained from the three individual western blots were quantified using Image $\mathrm{J}$ software to generate a bar graph of the relative difference in Hif-1 $\alpha$ protein expression levels. Data are presented as means + standard deviation. CXCR4, C-X-C chemokine receptor type 4; OA, osteoarthritis; HIF-1 $\alpha$, hypoxia-inducible factor $1 \alpha$.

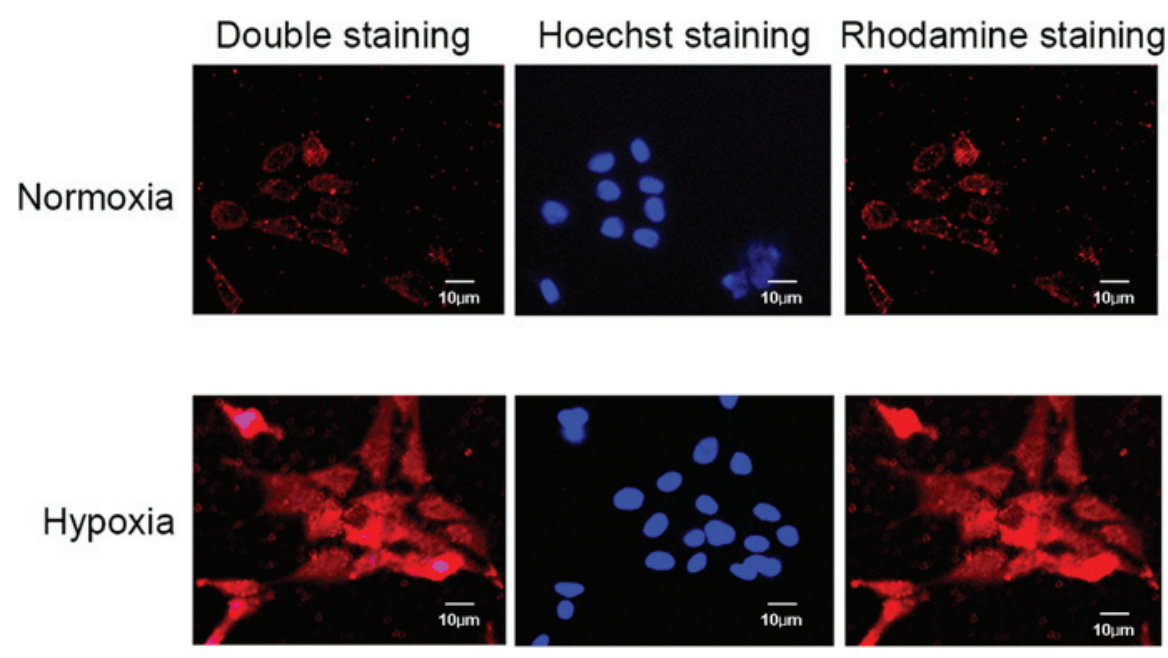

Figure 2. Immunofluorescence images of CXCR4 expression in osteoarthritis chondrocytes cultured under normoxic and hypoxic conditions (5 and $2 \%$ oxygen, respectively). Cells were stained with an anti-CXCR4 antibody (rhodamine, red) and with the nuclear binding dye, Hoechst two days later. Staining of CXCR4 was stronger in cells cultured under hypoxic conditions compared with those cultured in normoxic conditions. CXCR4, C-X-C chemokine receptor type 4.

following $48 \mathrm{~h}$ treatment with SDF-1 alone or SDF-1 plus AMD3100. Controls were cultured in the absence of SDF-1 and AMD3100. Runx 2 mRNA and protein expression levels were determined by RT-qPCR and western blotting, respectively. The results indicated that SDF-1 increased Runx2 mRNA $(\mathrm{P}=0.003)$ and protein expression levels, while the upregulation of Runx2 induced by SDF-1 was partially inhibited by AMD3100 ( $\mathrm{P}=0.04$; Fig. 4A and B).

SDF-1 increases GAG release in the conditioned media. GAG is an important structural component of proteoglycans and its release is a marker of proteoglycan catabolism (27). To further 
A

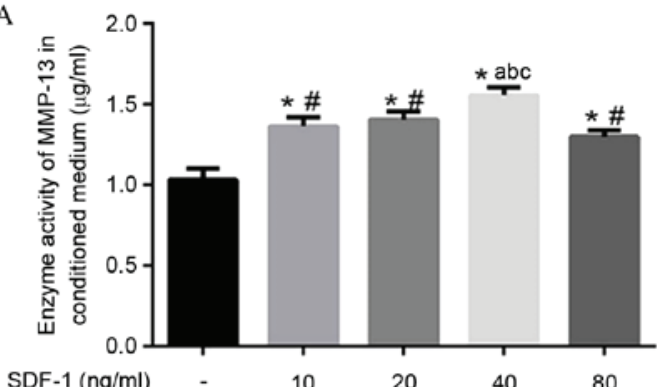

B

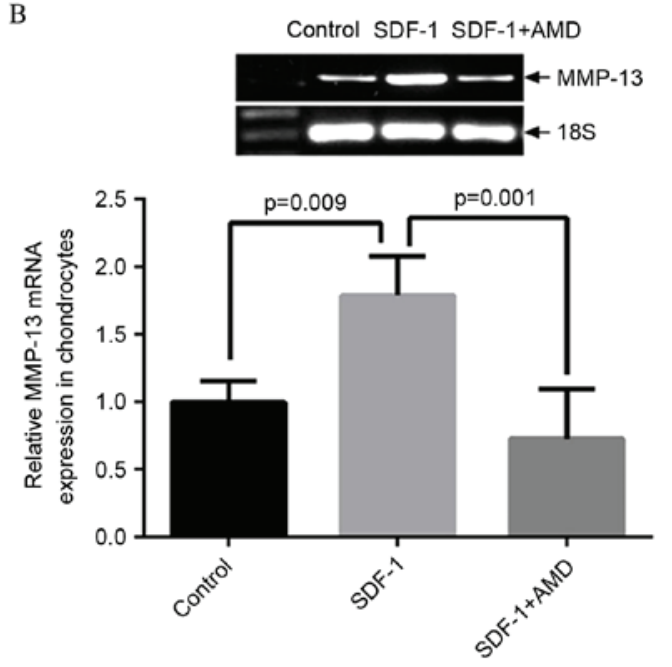

C

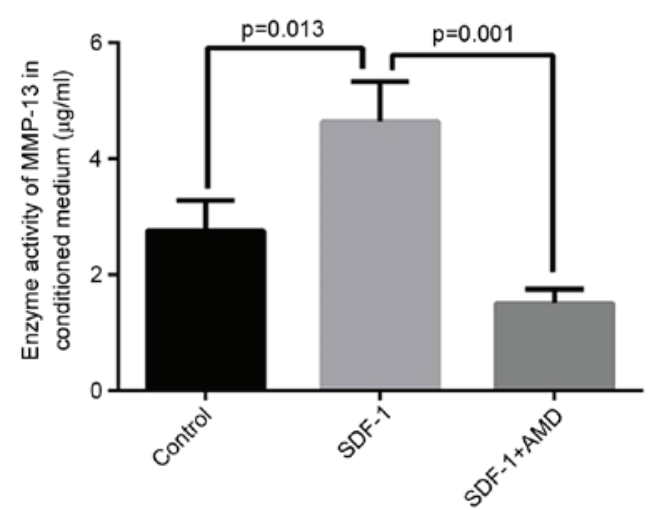

Figure 3. SDF-1/CXCR4 signaling pathway regulates MMP-13 expression and activity. (A) SDF-1 induced MMP-13 activity in a dose-dependent manner. Cells were treated with SDF-1 in serial dosages of 10, 20,40 and $80 \mathrm{ng} / \mathrm{ml}$ for two days and the conditioned media were collected for ELISA analysis. The increasing SDF-1 effect reached its highest point at the dosage of $40 \mathrm{ng} / \mathrm{ml}$. ${ }^{*} \mathrm{P}<0.05$ vs. control; ${ }^{~} \mathrm{P}<0.05$ vs. cells treated with $40 \mathrm{ng} / \mathrm{ml}$ SDF-1; ${ }^{\mathrm{a} P}<0.05$ vs. cells treated with $10 \mathrm{ng} / \mathrm{ml} \mathrm{SDF}-1 ;{ }^{b} \mathrm{P}<0.05$ vs. cells treated with $20 \mathrm{ng} / \mathrm{ml} \mathrm{SDF}-1 ;{ }^{\mathrm{C}} \mathrm{P}<0.05$ vs. cells treated with $80 \mathrm{ng} / \mathrm{ml}$ SDF-1. (B) Reverse transcription-quantitative polymerase chain reaction analysis of MMP-13 expression in osteoarthritis chondrocytes treated with SDF-1. SDF-1 significantly increased MMP-13 mRNA expression level in cells ( $\mathrm{P}=0.009)$, which was significantly reduced by blocking the SDF-1 pathway with AMD $3100(\mathrm{P}=0.001)$. (C) ELISA analysis of enzyme activity of MMP-13 within conditioned media. SDF-1 significantly increased the activity of MMP-13 $(\mathrm{P}=0.013)$, while a significant suppression of MMP-13 activity was observed by blocking the SDF-1 pathway with AMD 3100 ( $\mathrm{P}=0.001)$. SDF-1, stromal cell-derived factor-1; CXCR4, C-X-C chemokine receptor type 4; MMP-13, matrix metalloproteinase 13 .

examine whether SDF-1 causes cartilage matrix degradation, the concentration of GAG in the conditioned media of explants treated with SDF-1 was determined by spectrophotometry using
A
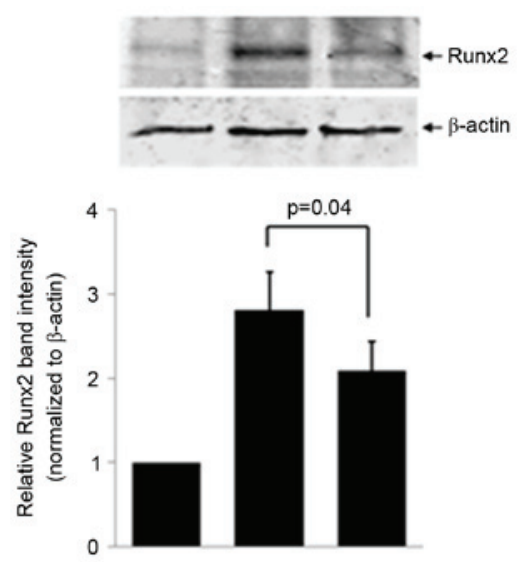

\begin{tabular}{|c|c|c|c|}
\hline SDF-1 (40 ng/mL) & - & + & + \\
\hline AMD3100 & - & - & + \\
\hline
\end{tabular}

B

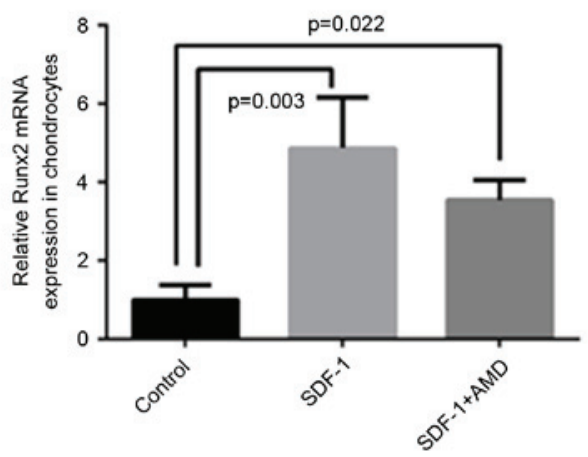

Figure 4. SDF-1/CXCR4 increases Runx2 expression levels. (A) Western blot analysis of Runx 2 and a bar graph of the relative difference in Runx 2 protein expression levels. The band intensities obtained from the three individual western blots were quantified using Image $\mathbf{J}$ software and indicated that SDF-1 treatment of osteoarthritis chondrocytes markedly increased Runx2 protein expression, while pretreatment of these cells with AMD3100 significantly reduced this effect. (B) Reverse transcription-quantitative polymerase chain reaction analysis of Runx2 indicated that SDF-1 significantly increased the expression of Runx2 while pretreatment of cells with AMD3100 significantly reduced this effect. Data are presented as means + standard deviation. SDF-1, stromal cell-derived factor-1; CXCR4, C-X-C chemokine receptor type 4; Runx2, runt-related transcription factor 2 .

a DMMB assay, in which bovine chondroitin sulfate served as a standard. The level of GAG was significantly higher in media collected from explants treated with SDF-1 vs. explants cultured in the absence of SDF-1 $(\mathrm{P}=0.001)$ or explants pretreated with AMD3100 prior to SDF-1 incubation ( $\mathrm{P}=0.004$; Fig. 5).

SDF-1 increases $I L-1 \beta$ release in the conditioned media. The level of IL-1 $\beta$ was significantly higher in media collected from explants treated with SDF-1 vs. explants cultured in the absence of SDF-1 $(\mathrm{P}=0.012)$ or explants treated with AMD3100 prior to SDF-1 incubation ( $\mathrm{P}=0.002$; Fig. 6).

\section{Discussion}

OA is a complex disease and its underlying pathological mechanisms remain to be elucidated. Genetic, biochemical 


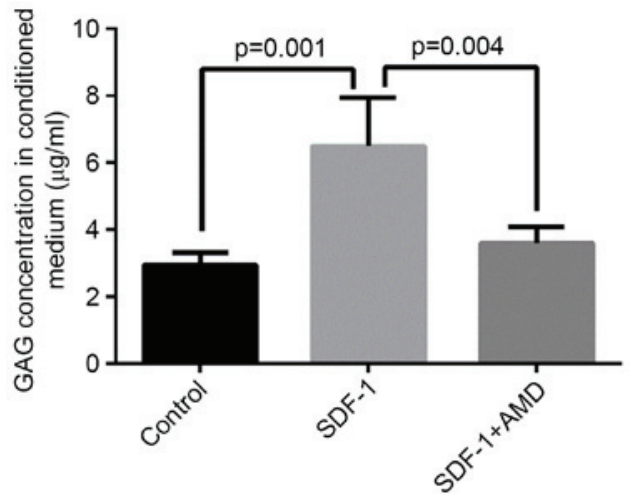

Figure 5. SDF-1-induced GAG release decreased in response to AMD3100 pretreatment in osteoarthritis cartilage explants. Free GAG content was significantly higher in media collected from explants treated with SDF-1 vs. explants without SDF-1 treatment or explants pre-treated with AMD3100 prior to SDF-1 incubation. This effect of SDF-1 was significantly reduced in the explants that were pretreated with AMD3100. Data are presented as means + standard deviation. SDF-1, stromal cell-derived factor-1; CXCR4, C-X-C chemokine receptor type 4; GAG, glycosaminoglycan.

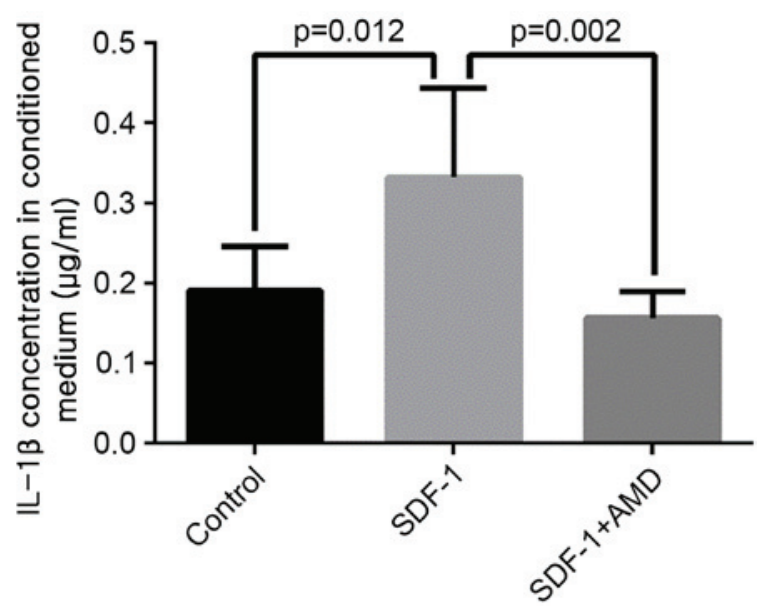

Figure 6. AMD3100 reduced SDF-1-induced IL-1 $\beta$ release into the conditioned media of cultured osteoarthritis cartilage explants. The expression level of IL-1 $\beta$ was significantly higher in media collected from explants treated with SDF-1 vs. explants without SDF-1 treatment or explants pre-treated with AMD3100 prior to SDF-1 incubation. Data are presented as means + standard deviation. SDF-1, stromal cell-derived factor-1; CXCR4, C-X-C chemokine receptor type 4; IL-1 $\beta$, interleukin-1 $\beta$.

and biomechanical factors all contribute to the pathogenesis of OA (28-30). It is well established that the inflammatory cytokines, IL-1 $\beta$ and TNF- $\alpha$ are closely associated with OA-induced cartilage degradation $(3,7)$. Previous studies have suggested that the SDF-1/CXCR4 signaling pathway is also an important facet of articular cartilage biology and may be involved in the pathogenesis of OA $(11,31)$. SDF-1 belongs to the CXC chemokine family and CXCR4 is a G-protein coupled receptor that, when activated by SDF-1, triggers a cascade of molecular events that ultimately regulate many important cellular functions, including chemotaxis, proliferation, apoptosis and differentiation (32). Increased expression of CXCR4 is a defining feature of hypertrophic chondrocytes (8). Furthermore, premature terminal differentiation of chondrocytes is indicative of OA pathogenesis as OA cartilage often consists of large zones of hypertrophic tissue $(33,34)$.
The aim of the present study was to investigate the role of CXCR4 activation in the context of OA chondrocyte and cartilage tissue catabolism. In the current study, cells were cultured under hypoxic conditions to mimic the physiological state of hypoxia, a defining characteristic of cartilage tissue, to examine how these conditions affect CXCR4 expression in OA chondrocytes. It was demonstrated that hypoxia significantly increases the CXCR4 expression level in human OA chondrocytes, which is consistent with the findings of a previous study in which hypoxia increased CXCR4 expression in a chondrosarcoma cell line (22). As it has been previously suggested that synovial fibroblasts exhibit increased SDF-1 production during OA conditions (9), the results of the present study suggest that the additional insult of hypoxia-induced CXCR4 elevation in chondrocytes may contribute to hyper-activation of the SDF-1/CXCR4 signaling pathway, thus favoring cartilage catabolism. To test this hypothesis, the effect of treating OA chondrocytes cultured in hypoxic conditions with SDF-1 was examined, and observed to increase MMP-13 mRNA expression and enzyme activity. This is consistent with a previous study, which demonstrated that SDF-1 increased MMP-3 and -13 levels in a dose-dependent manner, although this study was not conducted under hypoxic conditions (11). Increased MMP-13 production and activity is a canonical indicator of OA-induced cartilage catabolism, as it degrades type II collagen, a major building block of the cartilage ECM $(10,35,36)$. MMP-13 has been reported to be overexpressed in OA cartilage, and is a key inducer of events associated with OA $(6,20,37)$.

Consistent with previous findings, increased mRNA and protein expression levels of Runx2, a well-known regulator of MMP-13 (13,38-41), were observed in the present study in response to SDF-1 treatment of OA chondrocytes (13). Runx2 is an important transcription factor regulating cartilage maturation and bone formation $(42,43)$, and it has previously been linked to chondrocyte hypertrophy, which often occurs in OA. Notably, a heterozygous Runx2-deficient (Runx $2^{+/-}$) OA mouse model demonstrated decreased cartilage destruction, along with a reduction in MMP-13 expression (44). Furthermore, Runx2 directly upregulates MMP-13 by binding to a particular C-module-binding factor/nuclear matrix protein-2/osteoblastic cis-element 2 element in the promoter region of the MMP-13 gene $(12,45)$. The concomitant upregulation of Runx 2 and MMP-13 in the presence of SDF-1 suggests that SDF-1 signaling is a prerequisite for Runx2-regulated MMP-13 expression in OA chondrocytes, and that at least a component of the function of SDF-1/CXCR4 is exerted through Runx2-mediated regulation of MMP-13 expression. This is further substantiated by a recent study which demonstrated that knockdown of SDF-1 signaling in mesenchymal stem/progenitor cells undergoing chondrogenesis results in significant suppression of Runx2 expression (46).

In addition to increasing Runx 2 expression levels and MMP-13 activity under hypoxic conditions, SDF-1 increased IL-1 $\beta$ production and GAG release into the conditioned media of cartilage explants. This suggests that SDF-1 may induce mediators of OA-associated cartilage catabolism, and in this respect it may be functionally involved in OA pathogenesis. This is consistent with a previous study that reported elevated SDF-1 levels in the synovial fluid of OA patients (9). 
Furthermore, while it is reasonable to expect that the state of hypoxia that is inherent to cartilage tissue elevates CXCR4 expression in chondrocytes, the results of the present study demonstrate for the first time, to the best of our knowledge, that AMD3100 effectively prevents the aforementioned catabolic effects of SDF-1. AMD3100 is a specific inhibitor of CXCR4 that was first recognized for its potent activity against the HIV infection (47). A previous study identified AMD3100 to be effective in treating autoimmune collagen-induced arthritis in interferon $\gamma$ receptor-deficient DBA/1 mice (48). The present study reports the novel discovery that blocking CXCR4 using AMD3100 effectively reduces the expression of Runx2, inhibits MMP-13 activity and decreases GAG release under hypoxic conditions. The present study provides insight into the mechanism of OA pathogenesis, and suggests that the SDF-1/CXCR4 signaling pathway is hyper-activated in a hypoxic environment, such as the articular cartilage and induces cartilage matrix degradation via Runx2-mediated MMP-13 release. Furthermore, the present study has demonstrated for the first time, to the best of our knowledge, that SDF-1 stimulates the release of the inflammatory cytokine, IL-1 $\beta$ from OA cartilage explants; an effect that is abolished by the CXCR4 inhibitor, AMD3100. Based on the findings of the present study, blocking the SDF-1/CXCR4 signaling pathway by AMD3100 may potentially prevent OA progression.

Previous studies have demonstrated that animals tolerated AMD3100 well at a dose of $160 \mu \mathrm{g} /$ per day, a dose that has been reported safe in animals and humans (49-51). The severity of OA cartilage degeneration is attenuated by blocking SDF-1/CXCR4 signaling by continuous infusion of the CXCR4 blocker, AMD3100 via osmotic minipump, i.p (31). These data present a novel therapeutic target for the prevention and treatment of OA. However, the potential risk of intra-articular injection of AMD3100 has not been evaluated. Further research is required to evaluate the potential risk of intra-articular injection of AMD3100 and to establish a method of ensuring AMD3100 is released slowly.

In conclusion, the findings of the present study indicate that hypoxia upregulates CXCR4 expression in OA chondrocytes, that SDF-1/CXCR4 may be crucial in OA pathogenesis and that AMD3100 may abolish SDF-1-induced catabolic effects. Thus, blocking SDF-1 interaction with its receptor, CXCR4 may be applicable to developing chondro-protective therapy to treat inflammatory chemokine-induced arthritis. Further studies addressing these points are necessary to evaluate the therapeutic value of AMD3100 in large animal models of OA prior to clinical development.

\section{Acknowledgements}

The present study was supported by grants from the following: NIH/National Institute of Arthritis and Musculoskeletal and Skin Diseases (grant no. R01AR059142), NIH/National Center for Research Resources (grant no. P20RR024484), National Natural Science Foundation of China (grant nos. 81171676, 31271033 and 81572098), Shanxi Province Science and Technology Research Projects (grant no. 20150313012-6), The Second Hospital of Shanxi Medical University Doctor Funds (grant no. 20140406) and Research Project Supported by Shanxi Scholarship Council of China (grant no. 2016-122).
The content is solely the responsibility of the authors and does not necessarily represent the official view of the NIH. The authors gratefully acknowledge Dr Ericka M. Bueno, for help with the paper preparation and editorial services.

\section{References}

1. Arden N and Nevitt MC: Osteoarthritis: Epidemiology. Best Pract Res Clin Rheumatol 20: 3-25, 2006.

2. Miller ME, Rejeski WJ, Messier SP and Loeser RF: Modifiers of change in physical functioning in older adults with knee pain: The Observational Arthritis Study in Seniors (OASIS). Arthritis Rheum 45: 331-339, 2001.

3. Martel-Pelletier J, Boileau C, Pelletier JP and Roughley PJ: Cartilage in normal and osteoarthritis conditions. Best Pract Res Clin Rheumatol 22: 351-384, 2008

4. Aida Y, Maeno M, Suzuki N, Shiratsuchi H, Motohashi M and Matsumura $\mathrm{H}$ : The effect of IL-1beta on the expression of matrix metalloproteinases and tissue inhibitors of matrix metalloproteinases in human chondrocytes. Life Sci 77: 3210-3221, 2005.

5. Daheshia M and Yao JQ: The interleukin 1beta pathway in the pathogenesis of osteoarthritis. J Rheumatol 35: 2306-2312, 2008.

6. Mitchell PG, Magna HA, Reeves LM, Lopresti-Morrow LL, Yocum SA, Rosner PJ, Geoghegan KF and Hambor JE: Cloning, expression, and type II collagenolytic activity of matrix metalloproteinase-13 from human osteoarthritic cartilage. J Clin Invest 97: 761-768, 1996.

7. Jayasuriya CT, Goldring MB, Terek R and Chen Q: Matrilin-3 induction of IL-1 receptor antagonist is required for up-regulating collagen II and aggrecan and down-regulating ADAMTS-5 gene expression. Arthritis Res Ther 14: R197, 2012.

8. Wei L, Kanbe K, Lee M, Wei X, Pei M, Sun X, Terek R and Chen Q: Stimulation of chondrocyte hypertrophy by chemokine stromal cell-derived factor 1 in the chondro-osseous junction during endochondral bone formation. Dev Biol 341: 236-245, 2010.

9. Kanbe K, Takagishi K and Chen Q: Stimulation of matrix metalloprotease 3 release from human chondrocytes by the interaction of stromal cell-derived factor 1 and CXC chemokine receptor 4 . Arthritis Rheum 46: 130-137, 2002.

10. Wei L, Sun X, Kanbe K, Wang Z, Sun C, Terek R and Chen Q: Chondrocyte death induced by pathological concentration of chemokine stromal cell-derived factor-1. J Rheumatol 33: 1818-1826, 2006

11. Kanbe K, Takemura T, Takeuchi K, Chen Q, Takagishi K and Inoue K: Synovectomy reduces stromal-cell-derived factor-1 (SDF-1) which is involved in the destruction of cartilage in osteoarthritis and rheumatoid arthritis. J Bone Joint Surg Br 86: 296-300, 2004.

12. Jiménez MJ, Balbín M, López JM, Alvarez J, Komori T and López-Otín C: Collagenase 3 is a target of Cbfa1, a transcription factor of the runt gene family involved in bone formation. Mol Cell Biol 19: 4431-4442, 1999.

13. Wang X, Manner PA, Horner A, Shum L, Tuan RS and Nuckolls GH: Regulation of MMP-13 expression by RUNX2 and FGF2 in osteoarthritic cartilage. Osteoarthritis Cartilage 12: 963-973, 2004.

14. Zhu W, Boachie-Adjei O, Rawlins BA, Frenkel B, Boskey AL, Ivashkiv LB and Blobel CP: A novel regulatory role for stromal-derived factor-1 signaling in bone morphogenic protein-2 osteogenic differentiation of mesenchymal C2C12 cells. J Biol Chem 282: 18676-18685, 2007.

15. Lund-Olesen K: Oxygen tension in synovial fluids. Arthritis Rheum 13: 769-776, 1970.

16. Zhou S, Cui Z and Urban JP: Factors influencing the oxygen concentration gradient from the synovial surface of articular cartilage to the cartilage-bone interface: A modeling study. Arthritis Rheum 50: 3915-3924, 2004.

17. Koay EJ and Athanasiou KA: Hypoxic chondrogenic differentiation of human embryonic stem cells enhances cartilage protein synthesis and biomechanical functionality. Osteoarthritis Cartilage 16: 1450-1456, 2008.

18. Schioppa T, Uranchimeg B, Saccani A, Biswas SK, Doni A, Rapisarda A, Bernasconi S, Saccani S, Nebuloni M, Vago L, et al: Regulation of the chemokine receptor CXCR4 by hypoxia. J Exp Med 198: 1391-1402, 2003.

19. Staller P, Sulitkova J, Lisztwan J, Moch H, Oakeley EJ and Krek W: Chemokine receptor CXCR4 downregulated by von Hippel-Lindau tumour suppressor pVHL. Nature 425: 307-311, 2003. 
20. De Clercq E: The bicyclam AMD3100 story. Nat Rev Drug Discov 2: 581-587, 2003.

21. Pulsatelli L, Dolzani P, Piacentini A, Silvestri T, Ruggeri R, Gualtieri G, Meliconi R and Facchini A: Chemokine production by human chondrocytes. J Rheumatol 26: 1992-2001, 1999.

22. Sun X, Wei L, Chen Q and Terek RM: CXCR4/SDF1 mediate hypoxia induced chondrosarcoma cell invasion through ERK signaling and increased MMP1 expression. Mol Cancer 9: 17, 2010.

23. Sun X, Charbonneau C, Wei L, Yang W, Chen Q and Terek RM: CXCR4-targeted therapy inhibits VEGF expression and chondrosarcoma angiogenesis and metastasis. Mol Cancer Ther 12: 1163-1170, 2013

24. Farndale RW, Buttle DJ and Barrett AJ: Improved quantitation and discrimination of sulphated glycosaminoglycans by use of dimethylmethylene blue. Biochim Biophys Acta 883: 173-177, 1986.

25. Guan YJ, Yang X, Wei L and Chen Q: MiR-365: A mechanosensitive microRNA stimulates chondrocyte differentiation through targeting histone deacetylase 4. FASEB J 25: 4457-4466, 2011.

26. Livak KJ and Schmittgen TD: Analysis of relative gene expression data using real-time quantitative PCR and the 2(-Delta Delta C(T)) method. Methods 25: 402-408, 2001.

27. Lohmander S: Proteoglycans of joint cartilage. Structure, function, turnover and role as markers of joint disease. Baillieres Clin Rheumatol 2: 37-62, 1988.

28. Krasnokutsky S, Attur M, Palmer G, Samuels J and Abramson SB: Current concepts in the pathogenesis of osteoarthritis. Osteoarthritis Cartilage (16 Suppl 3): S1-S3, 2008.

29. Goldring MB: Osteoarthritis and cartilage: The role of cytokines. Curr Rheumatol Rep 2: 459-465, 2000.

30. Guilak F: Biomechanical factors in osteoarthritis. Best Pract Res Clin Rheumatol 25: 815-823, 2011.

31. Wei F, Moore DC, Li Y, Zhang G, Wei X, Lee JK and Wei L: Attenuation of osteoarthritis via blockade of the SDF-1/CXCR4 signaling pathway. Arthritis Res Ther 14: R177, 2012.

32. Ganju RK, Brubaker SA, Meyer J, Dutt P, Yang Y, Qin S, Newman W and Groopman JE: The alpha-chemokine, stromal cell-derived factor-1alpha, binds to the transmembrane G-protein-coupled CXCR-4 receptor and activates multiple signal transduction pathways. J Biol Chem 273: 23169-23175, 1998.

33. Dreier R: Hypertrophic differentiation of chondrocytes in osteoarthritis: The developmental aspect of degenerative joint disorders. Arthritis Res Ther 12: 216, 2010.

34. van der Kraan PM and van den Berg WB: Chondrocyte hypertrophy and osteoarthritis: Role in initiation and progression of cartilage degeneration? Osteoarthritis Cartilage 20: 223-232, 2012.

35. Massova I, Kotra LP, Fridman R and Mobashery S: Matrix metalloproteinases: Structures, evolution, and diversification. FASEB J 12: 1075-1095, 1998.

36. Knäuper V, Will H, López-Otin C, Smith B, Atkinson SJ, Stanton H, Hembry RM and Murphy G: Cellular mechanisms for human procollagenase-3 (MMP-13) activation. Evidence that MT1-MMP (MMP-14) and gelatinase a (MMP-2) are able to generate active enzyme. J Biol Chem 271: 17124-17131, 1996.

37. Billinghurst RC, Dahlberg L, Ionescu M, Reiner A, Bourne R, Rorabeck C, Mitchell P, Hambor J, Diekmann O, Tschesche H, et al: Enhanced cleavage of type II collagen by collagenases in osteoarthritic articular cartilage. J Clin Invest 99: 1534-1545, 1997.
38. Enomoto $\mathrm{H}$, Enomoto-Iwamoto $\mathrm{M}$, Iwamoto $\mathrm{M}$, Nomura $\mathrm{S}$, Himeno M, Kitamura Y, Kishimoto T and Komori T: Cbfal is a positive regulatory factor in chondrocyte maturation. J Biol Chem 275: 8695-8702, 2000.

39. Ueta C, Iwamoto M, Kanatani N, Yoshida C, Liu Y, Enomoto-Iwamoto M, Ohmori T, Enomoto H, Nakata $\mathrm{K}$, Takada K, et al: Skeletal malformations caused by overexpression of Cbfa1 or its dominant negative form in chondrocytes. J Cell Biol 153: 87-100, 2001.

40. Kim IS, Otto F, Zabel B and Mundlos S: Regulation of chondrocyte differentiation by Cbfa1. Mech Dev 80: 159-170, 1999.

41. Zheng Q, Zhou G, Morello R, Chen Y, Garcia-Rojas X and Lee B: Type $X$ collagen gene regulation by Runx 2 contributes directly to its hypertrophic chondrocyte-specific expression in vivo. J Cell Biol 162: 833-842, 2003.

42. Ducy P, Zhang R, Geoffroy V, Ridall AL and Karsenty G: Osf2/ Cbfa1: A transcriptional activator of osteoblast differentiation. Cell 89: 747-754, 1997.

43. Karsenty G and Wagner EF: Reaching a genetic and molecular understanding of skeletal development. Dev Cell 2: 389-406, 2002.

44. Kamekura S, Kawasaki Y, Hoshi K, Shimoaka T, Chikuda H, Maruyama Z, Komori T, Sato S, Takeda S, Karsenty G, et al: Contribution of runt-related transcription factor 2 to the pathogenesis of osteoarthritis in mice after induction of knee joint instability. Arthritis Rheum 54: 2462-2470, 2006.

45. Pendás AM, Balbín M, Llano E, Jiménez MG and López-Otín C: Structural analysis and promoter characterization of the human collagenase-3 gene (MMP13). Genomics 40: 222-233, 1997.

46. Guang LG, Boskey AL and Zhu W: Regulatory role of stromal cell-derived factor-1 in bone morphogenetic protein-2-induced chondrogenic differentiation in vitro. Int J Biochem Cell Biol 44: 1825-1833, 2012.

47. De Clercq E, Yamamoto N, Pauwels R, Baba M, Schols D, Nakashima H, Balzarini J, Debyser Z, Murrer BA, Schwartz D, et al: Potent and selective inhibition of human immunodeficiency virus (HIV)-1 and HIV-2 replication by a class of bicyclams interacting with a viral uncoating event. Proc Natl Acad Sci USA 89: 5286-5290, 1992.

48. Ma Q, Jones D, Borghesani PR, Segal RA, Nagasawa T, Kishimoto T, Bronson RT and Springer TA: Impaired B-lymphopoiesis, myelopoiesis, and derailed cerebellar neuron migration in CXCR4- and SDF-1-deficient mice. Proc Natl Acad Sci USA 95: 9448-9453, 1998.

49. Matthys P, Hatse S, Vermeire K, Wuyts A, Bridger G, Henson GW, De Clercq E, Billiau A and Schols D: AMD3100, a potent and specific antagonist of the stromal cell-derived factor-1 chemokine receptor CXCR4, inhibits autoimmune joint inflammation in IFN-gamma receptor-deficient mice. J Immunol 167: 4686-4692, 2001.

50. Hendrix CW, Flexner C, MacFarland RT, Giandomenico C, Fuchs EJ, Redpath E, Bridger $G$ and Henson GW: Pharmacokinetics and safety of AMD-3100, a novel antagonist of the CXCR-4 chemokine receptor, in human volunteers. Antimicrob Agents Chemother 44: 1667-1673, 2000.

51. Hendrix CW, Collier AC, Lederman MM, Schols D, Pollard RB, Brown S, Jackson JB, Coombs RW, Glesby MJ, Flexner CW, et al: Safety, pharmacokinetics, and antiviral activity of AMD3100, a selective CXCR4 receptor inhibitor, in HIV-1 infection. J Acquir Immune Defic Syndr 37: 1253-1262, 2004. 